\title{
Reviewing the Potential Vectors and Hosts of African Swine Fever Virus Transmission in the United States
}

\author{
Andrew J. Golnar, ${ }^{1}$ Estelle Martin,, Jillian D. Wormington, ${ }^{2}$ Rebekah C. Kading, ${ }^{3}$ \\ Pete D. Teel, ${ }^{1}$ Sarah A. Hamer, ${ }^{2}$ and Gabriel L. Hamer ${ }^{1}$
}

\begin{abstract}
African swine fever virus (ASFV) continues to threaten global animal health and agricultural biosecurity. Mitigating the establishment of ASFV in the United States (U.S.) is contingent on (1) the identification of arthropod vectors and vertebrate hosts that are capable of viral maintenance and transmission in the U.S. and (2) knowledge of vector-host associations that may permit transmission. We aggregated data on vector competence, host competence and tick-host associations by systematic review of published articles and collection records to identify species that may support the invasion of ASFV in the U.S. Three species of competent soft ticks occur in the U.S., Ornithodoros coriaceus, Ornithodoros turicata, and Ornithodoros puertoricensis, however, vector competence for the majority of soft ticks in the U.S. remains unknown. Three species of competent vertebrate hosts currently occur in the U.S.: domestic pigs (Sus scrofa domesticus), feral hogs (Sus scrofa), and common warthogs (Phacochoerus africanus). Hierarchical hazard categories based on vector competence, tick-host contact rates, and vector abundance were used to semiquantitatively rank U.S. soft tick species by their relative risk for contributing to ASFV transmission to identify which soft tick species are a priority for future studies. High-risk vector and host species identified in this study can be used to focus ASFV risk assessments in the U.S., guide targeted surveillance and control strategies, and proactively prepare for an ASFV incursion event. Results indicate $O$. coriaceus, $O$. turicata, and $O$. puertoricensis demonstrate the highest relative risk for contributing to ASFV transmission in the U.S., however, many gaps in knowledge exist preventing the full evaluation of at least 30 soft tick species in the U.S. Further study is required to identify soft tick vectors that interact with feral swine populations, elucidate vector competence, and further understand the biology of soft tick species.
\end{abstract}

Keywords: Argasidae, vector competence, host competence, swine, African swine fever

\section{Introduction}

A FRICAN SWINE FEVER VIRUS (ASFV) is a DNA virus (Family: Asfarviridae, Genus: Asfivirus) associated with severe epizootics and pathology in domestic pig populations. As such, viral outbreaks are accompanied with serious socioeconomic consequences and should be proactively managed (Fasina et al. 2012).

The virus was first described in Kenya in 1921 (Montgomery 1921) and has emerged in regions of Africa, Europe, the Caribbean, and South America facilitated by growing international trade networks and swill practices that expose swine to infection (Wooldridge et al. 2006, Costard et al. 2013, Roelandt et al. 2017). Although the virus has been extirpated at considerable cost from regions, including Brazil, Cuba, and Spain, the virus remains endemic in subSaharan Africa, Madagascar, and Sardinia (Italy) and in the last decade has caused multiple outbreaks in the Republic of Georgia, Azerbaijan, Armenia, Ukraine, Belarus, the Russian Federation, Lithuania, Poland, Estonia, Latvia, and most

\footnotetext{
${ }^{1}$ Texas A\&M AgriLife Research, Department of Entomology, Texas A\&M University, College Station, Texas.

${ }_{3}^{2}$ Department of Veterinary Integrative Biosciences, Texas A\&M University, College Station, Texas.

${ }^{3}$ Department of Microbiology, Immunology \& Pathology, Colorado State University, Fort Collins, Colorado.
}

(C) Andrew J. Golnar et al. 2019; Published by Mary Ann Liebert, Inc. This Open Access article is distributed under the terms of the Creative Commons License (http://creativecommons.org/licenses/by/4.0), which permits unrestricted use, distribution, and reproduction in any medium, provided the original work is properly cited. 
recently in the Luxembourg province of Belgium and the Anhui, Heilongjiang, Henan, Jilin, Liaoning, Jiangsu, Zhejiang provinces of China, and the Inner Mongolia Autonomous Region of China (Fernandez and Williams 1980, Arias and Sánchez-Vizcaíno 2002, Moura et al. 2010, Guinat et al. 2016a, Mur et al. 2016, Roelandt et al. 2017, ProMED-mail 2018).

The dynamics of ASFV transmission are complex and include several soft tick species of the Ornithodoros genus (Acari: Argasidae) and wild and domestic vertebrates from the family Suidae. As a DNA virus, ASFV is extremely persistent in its environment, such that contaminated secretions (blood, feces, urine, mucus) and fomites (vehicles, equipment) function as modes of transmission, in addition to vector-borne and aerosol transmission. Although vaccine technologies are in development, there is currently no vaccine or effective antiviral treatment. Disease management broadly aims to restrict animal movement, reduce contact between swine populations (domestic and wild), improve sanitation, and rapidly cull infected animals (Costard et al. 2009).

Although the roles for soft tick vectors and vertebrate hosts in disease maintanance, amplification, and spillover are diverse, a common feature of transmission is that the introduction of the virus to domestic populations results in severe epizootics, high case mortality, and disastrous economic consequences (Arias and Sánchez-Vizcaíno 2002, Moura et al. 2010, Fasina et al. 2012, Gogin et al. 2013, Brown and Bevins 2018). In the Russian Federation alone it is estimated that ASFV resulted in the loss of 800,000 pigs and between 833 million and 1.25 billion U.S. dollars since the virus was introduced in 2007 (United States Department of Agriculture 2017). As of October 6, 2018, just over 2 months following the initial report of ASFV in China (August 3, 2018), over 90,000 pigs have been culled in an effort to control the spread of ASFV (ProMED-mail 2018).

Due to the clear socioeconomic impacts of ASFV and a clear propensity to spread, ASFV is a pressing threat to global agricultural stability, especially for countries like China, which contains more than half of the world's pig population (Sanchez-Cordón et al. 2018). More than ever, it is important for countries with significant swine production to proactively prepare for an ASFV incursion event.

In endemic regions of Africa, ASFV is maintained in a sylvatic cycle among desert warthogs (Phacochoerus aethiopicus) and Ornithodoros tick species, including Ornithodoros moubata and Ornithodoros porcinus porcinus; however, bushpigs (Potamochoerus porcus) and giant forest hogs (Hylochoerus meinertzhageni) can also function as viral reservoirs. Although ASFV can be horizontally transmitted between swine (Guinat et al. 2016b, Davies et al. 2017), transmission from warthogs to domestic pigs is rare, suggesting soft tick vectors that inhabit pigsties, such as Ornithodoros porcinus domesticus, are important agents of viral spillover into domestic swine populations (Boinas et al. 2011).

Similarly, in regions of Europe, ASFV persists in a sylvatic cycle among wild boars (Sus scrofa) and the soft tick vector Ornithodoros erraticus (Jori and Bastos 2009). However, unlike the African system, recent reports posit that ASFV may persist among wild and domestic pig populations in the absence of soft tick vectors presumably through horizontal transmission (Pietschmann et al. 2016).
The ability to invade and persist without competent soft tick vectors further emphasizes the risk of ASFV to global swine populations and emphasizes a need to implement proactive disease management strategies (Guinat et al. 2014, 2016a, 2016b, Pietschmann et al. 2016). These data are particularly concerning to the agricultural security of the United States (U.S.), as the U.S. is one of the largest global swine industries and is now surrounded by an invasive population of over 6 million feral swine (S. scrofa) present in at least 35 states (Brown and Bevins 2018).

In anticipation of viral emergence in the U.S., agencies charged with mitigating viral invasion must focus on preventing introduction events, enhancing outbreak detection and preparing response strategies. The objective of this article is to systematically review and synthesize published vector competence data, vertebrate competence data, and tick-host association data to identify and rank tick and vertebrate species predicted to be important to the transmission of ASFV in the U.S. Results are intended to help prioritize future research on ASFV and protect health and economic stability in the U.S. by informing proactive prevention, detection, and response strategies.

\section{Methods}

\section{Vector competence}

Vector competence is the ability of an arthropod to acquire, support replication of, and transmit a pathogen to a susceptible vertebrate host. To identify competent vectors of ASFV, we retrieved articles written in the English language from the search engines Web of Science, NCBI's PubMed, and Science Direct with the search term "African swine fever virus."

After reviewing abstracts, data from experimental studies in which vectors were exposed to ASFV were extracted and aggregated in a database for review. Data on arthropod species, virus strain, infectious dose, route of exposure, viremia, sample size, incubation period, temperature, infection rate, estimated transmission rates, viral multiplication, viral persistence, and transmission routes were recorded when available. We also recorded whether studies documented transtadial transmission (between arthropod life stages) and transovarial transmission (from adult female to offspring). Literature was identified and reviewed between March 2017 and March 2018. All soft tick species throughout the entire study are referred to using the authority designated by Guglielmone et al. (2010).

\section{Host competence}

Host competence (also known as reservoir competence) is the assessment of an animal's ability to contribute to pathogen amplification and persistence and is a useful concept for judging the importance of different hosts to pathogen transmission (Komar et al. 2003, Golnar et al. 2014, Gervasi et al. 2015). In vector-borne disease systems, the role a vertebrate plays in amplifying a pathogen is often estimated based on the infectiousness of the host to a feeding vector and the frequency of contacts between infectious hosts and competent vectors (Kilpatrick et al. 2006, Hamer et al. 2009). Host infectiousness is often assumed to reflect the magnitude and duration of host parasitemia (defined broadly to include circulating titers of viral pathogens) observed during experimental infections (Komar et al. 2003). 
In vector-borne disease systems, host parasitemia has been strongly associated with vector infection, vector dissemination, and vector transmission rates (Ewald 1987, Komar et al. 2003). The magnitude and duration of host parasitemia is also likely to provide a strong indicator of host infectiousness for pathogens transmitted between vertebrate hosts (Ewald 1987). Therefore, the review of vertebrate viremia profiles after ASFV infection provides a useful framework for assessing host competence.

Experimental transmission studies that documented viremic titers in vertebrate hosts written in English were identified and aggregated using similar search and inclusion criteria as those stated above. Studies were identified using Web of Science, PubMed, and Science Direct with the search term "African swine fever virus." Titles and abstracts were reviewed to identify studies that exposed vertebrate hosts to ASFV and temporally quantify titers postinfection.

When available, vertebrate species, viral lineage, infectious dose, route of inoculation, and daily viremia postinfection were extracted and aggregated into a database for review. These data were used to understand the magnitude and duration of infection in different hosts by comparing viremia profiles over time. Although the viremic response in vertebrate hosts is expected to vary by viral strain, exposure dose, and route of inoculation, low sample sizes, nonstandardized methods, and the early termination of studies by euthanasia provided barriers to further statistical evaluation of these factors. In many cases, ASFV strains are genetically altered for various experimental questions, although genetically modified strains were excluded in this analysis, unaltered control ASFV isolates from the same studies were included.

\section{Vector-host association}

The spatial distribution of soft tick species in the U.S. and contact rates between soft tick vectors and vertebrate hosts were estimated using a database provided by the U.S. National Tick Collection, Georgia Southern University, Statesboro, GA. Specifically, records between years 1891 and 2004 were aggregated based on the number of unique collections of each soft tick species that were recorded to be associated with different mammalian orders (Rodentia, Cingulata, Carnivora, Chiroptera, Lagomorpha, Artiodactyla, Perissodactyla, and Primates). Reptilian and Avian taxa were aggregated at the taxonomic resolution of class.

Although the number of ticks collected in each entry were variable (range from 1 to $>2000$ individuals), each entry for tick-host pairs was counted as one data point. It is important to note that soft tick species are often nidicolous and are frequently not collected directly from vertebrate hosts. For this analysis, we rely on the explicit assumption that all documented associations in the database are accurate.

\section{Soft tick risk index}

To inform policy and future research directions, soft tick vectors in the U.S. were semiquantitatively ranked based on six hazard categories for which each tick species was assigned to the lowest (most severe) hazard level to which it qualified.

To participate in ASFV transmission, vectors must be competent, therefore vectors established to be competent were assigned a level 1 hazard (categorical value), which is considered the most severe. A vector's role in viral maintenance and amplification also depends on contact rates with competent hosts, therefore, tick association with the taxonomic family Suidae represents a level 2 hazard (continuous value) and tick association with the taxonomic order Artiodactyla is a level 3 hazard (continuous value). Because generalist feeding patterns on mammalian hosts may predict opportunistic contacts between soft tick vectors and swine, the number of mammalian hosts associated with a soft tick vector was defined as a level 4 hazard (continuous value).

Additionally, vector abundance is an important factor in assessing the relative risk of a tick vector for contributing to ASFV transmission in the U.S. As such, vector abundance was defined as a level 5 hazard (continuous value) and was estimated based on the number of U.S. counties, where ticks occur gathered from the U.S. National Tick Collection and the Global Biodiversity Information Facility (GBIF.org. 2018). Additionally, the number of collections reported to the U.S. National Tick Collection was utilized as an additional metric of vector abundance and was defined as a level 6 hazard (continuous value). The relative risk of a tick vector for contributing to ASFV transmission in the U.S. was determined by sorting the tick species by the defined hazard levels.

\section{Results}

Literature search identified 170 studies through Web of Science, PubMed, and Science Direct that were published between 1966 and 2017. These articles covered topics, including ASFV epidemiology, viral ecology, disease management, vaccine development, controlled laboratory experiments, pathogenesis, mathematical biology, and subject reviews.

From these studies, 15 published studies were identified that provided data on vector competence (Wilkinson et al. 1981, McVicar 1984, Villeda et al. 1993, Ramiroibanez et al. 1995, Anderson et al. 1998, Argilaguet et al. 2012, Karalyan et al. 2012, Guinat et al. 2014, O'Donnell et al. 2015a, 2015b, 2016, Carlson et al. 2016, Sanford et al. 2016, Popescu et al. 2017, Basto et al. 2006a) and 27 studies provided data to assess host competence (Wilkinson and Donaldson 1977, Wilkinson et al. 1977, 1981, Thomson et al. 1980, McVicar 1984, Knudsen and Genovesi 1987, Genovesi et al. 1988, Villeda et al. 1993, Ramiroibanez et al. 1995, Anderson et al. 1998, Argilaguet et al. 2012, Ferreira et al. 2012, 2013, Karalyan et al. 2012, de Carvalho Ferreira et al. 2013, Guinat et al. 2014, Nieto-Pelegrin et al. 2015, O'Donnell et al. 2015a, 2015b, 2016, Pietschmann et al. 2015, Carlson et al. 2016, Reis et al. 2016, Sanford et al. 2016, Gallardo et al. 2017, Popescu et al. 2017, Post et al. 2017).

A total of 128 published articles identified during the literature search were not included because they did not provide quantitative data useful for evaluating vector or host competence (citations not provided).

\section{Vector competence}

Globally, nine soft tick species (Ornithodoros marocanus, Ornithodoros puertoricensis, Ornithodoros coriaceus, O. moubata porcinus, O. erraticus, O. moubata complex, Ornithodoros turicata, Ornithodoros savignyi, Ornithodoros parkeri), four hard tick species (Dermacentor reticulatus, 


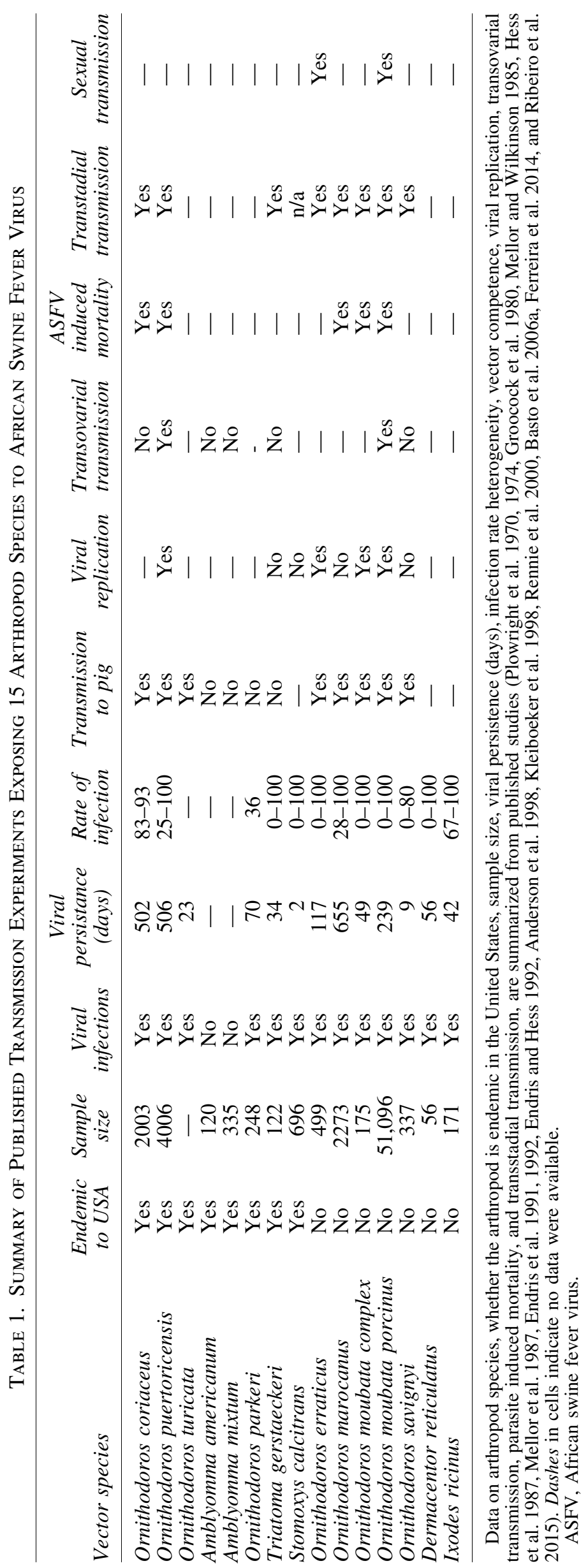


Ixodes ricinus, Amblyomma americanum, Amblyomma mixtum), and two insect species (Triatoma gerstaeckeri and Stomoxys calcitrans) have been evaluated for ASFV transmission competency.

Following engorgement on infectious blood, ASFV was detected in all vectors, except the hard ticks, A. americanum and $A$. mixtum. Infection prevalence was extremely variable by tick species, ranging between $0 \%$ and $100 \%$ within multiple species (Table 1).

Evidence from vector competence studies demonstrate that eight taxa-O. marocanus, O. puertoricensis, $O$. coriaceus, O. moubata porcinus, O. erraticus, O. moubata complex, $O$. turicata, and $O$. savignyi-are competent vectors. Three of these species-O. coriaceus, O. turicata, and $O$. puertoricensis-exist in the U.S. The U.S. vectors $O$. parkeri, $T$. gerstaeckeri, A. americanum, and A. mixtum are considered incompetent hosts (Table 1). Infection by ASFV was detected in the U.S. vectors, O. parkeri, T. gerstaeckeri, and S. calcitrans, however, transmission of ASFV from $O$. parkeri and $T$. gerstaeckeri to a susceptible host failed. Transmission of ASFV from $S$. calcitrans to a susceptible host was not tested. Infection of ASFV was not detected in the hard ticks A. americanum and A. mixtum (Table 1).

Transmission studies demonstrate ASFV can be detected up to 655 days postinfection in $O$. marocanus and remain infectious for at least 588 days (Table 1). Viral replication of ASFV was reported in $O$. puertoricensis, O. moubata porcinus, and O. moubata, whereas a failure of replication of ASFV was reported in $O$. marocanus, $O$. savignyi, $T$. gerstaeckeri, and S. calcitrans (Table 1).

Transovarial transmission was documented in $O$. puertoricensis and $O$. moubata porcinus, but not in $O$. coriaceus, O. savignyi, T. gerstaeckeri, A. americanum, or A. mixtum (Table 1). Transstadial transmission was observed in O. marocanus, O. puertoricensis, O. moubata, O. coriaceus, O. moubata porcinus, O. erraticus, O. moubata complex, O. savignyi, and T. gerstaeckeri (Table 1). Sexual transmission between vectors was observed in $O$. moubata porcinus and O. erraticus (Table 1). Vector mortality due to ASFV infection was documented in $O$. marocanus, O. puertoricensis, and $O$. coriaceus (Table 1$)$.

\section{Host competence}

Through methods of literature review, all species of vertebrate hosts exposed to ASFV were in the taxonomic family Suidae (bushpigs, warthogs, feral pigs, and domestic pigs). No studies included vertebrates outside of the Suidae family. Studies quantified viral titers postinfection with Tissue Culture Inoculation Dose $50\left(\mathrm{TCID}_{50}\right)$, cytopathic effect (CPE), quantitative PCR, CPE, hemadsorption in $50 \%$ of inoculated cells $\left(\mathrm{HAD}_{50}\right)$, and by hemagglutination assay (HA). Viremia profiles for 77 hosts (5 warthogs, 4 bushpigs, 68 domestic pigs) quantified by $\log _{10} \mathrm{HAD}_{50} / \mathrm{mL}$ (Fig. 1) illustrate that domestic pigs produce the highest viremic titers $\left(8.8 \log _{10}\right.$ $\mathrm{HAD}_{50} / \mathrm{mL}$ on day 6 P.I), followed by bushpigs $\left(5.3 \log _{10}\right.$ $\mathrm{HAD}_{50} / \mathrm{mL}$ on day 21 P.I $)$ and warthogs $\left(4.3 \log _{10} \mathrm{HAD}_{50} / \mathrm{mL}\right.$ day 35 P.I.).

Multiple warthogs were documented to have low viremia levels for up to 35 days P.I. $\left(<4.3 \log _{10} \mathrm{HAD}_{50} / \mathrm{mL}\right.$ ) (Anderson et al. 1998) (Fig. 1). Virions were documented in a bushpig for up to 70 days $\left(2.1 \log _{10} \mathrm{HAD}_{50} / \mathrm{mL}\right.$ ) (Anderson et al. 1998) (Fig. 1) and in domestic swine up to 40 days (4.2 1 $\log _{10}$ TCID $50 / \mathrm{mL}$ ) (not shown in Fig. 1) (Ferreira et al. 2012). It is important to note that animals infected with ASFV are often euthanized following IACUC-approved protocols suggesting that most estimates of viral persistence may be underestimations (Galindo-Cardiel et al. 2013).

\section{Tick-host association}

The US National Tick Collection database contained records in the U.S. from 1891 to 2004 and documented the collection of 31,793 soft ticks, from 39 tick species, by more than 450 different individuals. Soft ticks were associated with swine in only one collection record: $O$. coriaceus ticks were documented to be associated with $S$. scrofa in Sonoma County, California in 1995. Four soft tick species were reported to be associated with nine different genera in the order Artiodactyla on 202 occasions.

Based on documented host associations, species associated with Artiodactyla were Otobius megnini $(n=190)$, O. coriaceus $(n=11)$, O. turicata $(n=1)$, and O. parkeri. $(n=1)$ (Fig. 2). Soft ticks that were associated with mammalian hosts include: O. parkeri $(n=204)$, O. megnini $(n=190)$, O. turicata $(n=62)$, Ornithodoros kelleyi $(n=55)$, Otobius lagophilus (50), Ornithodoros sparnus $(n=49)$, Ornithodoros talaje $(n=42)$, Ornithodoros yumatensis $(n=30)$, O. coriaceus $(n=19)$, Ornithodoros stageri $(n=17)$, Ornithodoros hermsi $(n=12)$, Ornithodoros dyari $(n=5)$, Ornithodoros rossi $(n=5)$, Ornithodoros concanensis $(n=4)$, Argas cooleyi $(n=5)$, Argas sanchezi $(n=2)$, Ornithodoros dugesi $(n=2)$, Ornithodoros brasiliensis $(n=1)$, Ornithodoros hasei $(n=1)$, and Ornithodoros peropteryx $(n=1)$ (Fig. 3).

Soft ticks from the genus Argas were generally associated with vertebrates from the Avian class (Fig. 3).

\section{Soft tick risk index}

Vector competence and contact rates determined by host associations were used to rank which vectors would most likely be involved in ASFV transmission in the U.S.

$O$. coriaceus was ranked as the vector with the highest relative risk in the U.S. based on the hazard of vector competence and the hazardous associations with swine (Table 2). $O$. turicata was ranked as the vector with the second highest relative risk based on the hazard of vector competence and the hazardous associations with the vertebrate order Artiodactyla (Table 2, Figs. 2 and 3). O. puertoricensis was ranked as the vector with the third highest relative risk based on the hazard of vector competence (Table 2). O. megnini was ranked as the vector with the fourth highest relative risk based on their hazardous associations with hosts in the order Artiodactyla, however, the vector competence of this species remains unknown (Table 2).

Based on no demonstrated hazard of ASFV vector competence or no hazardous associations with Artiodactyla hosts, 18 tick species were identified as low risk (Table 2). Besides Ornithodoros parkeri, the vector competence for all these species remains unknown. $O$. parkeri demonstrated no ability to transmit ASFV during a vector competence study and is therefore estimated to be low risk.

Finally, 16 soft tick species were classified to be of unknown risk. These species have no vector competence data 


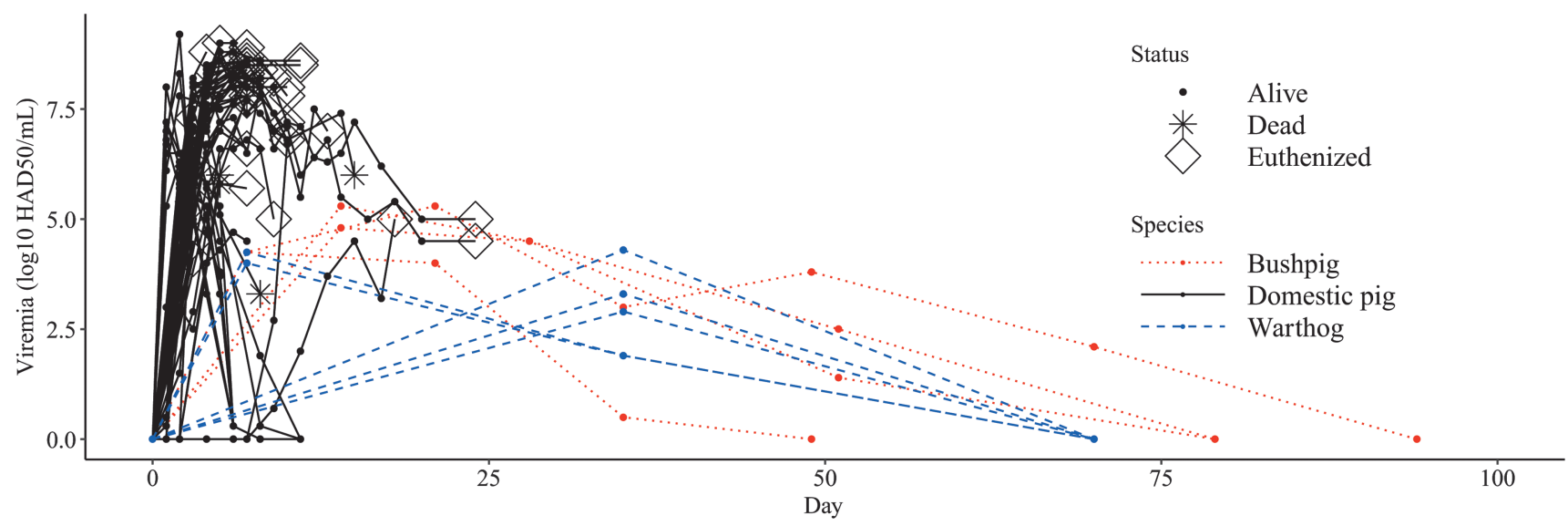

FIG. 1. Viremia profile of vertebrates exposed to African swine fever virus. Viremia profiles of three vertebrate species exposed to African swine fever virus through intramuscular inoculation, intranasal infection, direct and indirect exposure between infectious pigs is visualized from 14 studies that documented viremia with $\mathrm{HAD}_{50}$ and HA (Wilkinson et al. 1981, McVicar 1984, Villeda et al. 1993, Ramiroibanez et al. 1995, Anderson et al. 1998, Argilaguet et al. 2012, Karalyan et al. 2012, Guinat et al. 2014, O’Donnell et al. 2015a, 2015b, 2016, Carlson et al. 2016, Sanford et al. 2016, Popescu et al. 2017). Studies quantified viral titers postinfection with $\mathrm{HAD}_{50}$ and $\mathrm{HA}$, which are assumed to be equivalent measures of viral titer (Johnston et al. 1992). Vertebrate infectiousness depends on the magnitude of infection ( $y$-axis) and the duration of infection ( $x$-axis). Time series data on viremia titers ended when animals cleared infection $\left(0.0 \log _{10} \mathrm{HAD}_{50} / \mathrm{mL}\right)$, animals died from infection $(*)$, or were euthanized $(\diamond)$. HA, Hemagglutinin Assay; HAD ${ }_{50}$, Hemadsorption in $50 \%$ of inoculated cells.

and limited host association data $(<10$ documented associations with vertebrate hosts), which prevent hazard determination. Therefore, it is inconclusive whether these vectors may contact swine or contribute to ASFV amplification or maintenance (Table 2).

\section{Discussion}

Animal health and agricultural security are significantly threatened by the propensity of ASFV to disperse and invade populations of swine worldwide (Costard et al. 2009, Roelandt et al. 2017, Brown and Bevins 2018). Accordingly, strategies to prevent and mitigate the invasion and establishment of ASFV in the U.S. must identify (1) which U.S. vectors and hosts are capable of contributing to viral transmission and maintenance and (2) understanding contact rates between competent vectors with competent hosts. In this study, we aggregate vector competence data, host compe- tence data, tick prevalence, and tick-host association data to prioritize species that may play an important role in transmission and maintenance of ASFV should it arrive in the U.S.

Vector competence studies demonstrate that a variety of arthropods are susceptible to infection by ASFV. Mechanical transmission of the virus was observed in stable flies (S. calcitrans) (Mellor et al. 1987), but its role in endemic or epidemic transmission scenarios remains unknown. Furthermore, one experimental study demonstrated that ASFV may persist in kissing bugs (Family: Reduviidae, Subfamily: Triatominae) indicating host infection after accidental or the intentional ingestion of an infectious triatomine insect may be a potential mechanism of ASFV transmission, as observed in the Chagas disease system (Roellig et al. 2009, Pietschmann et al. 2015). However, whether domestic or wild swine ingest triatomine bugs or whether triatomine bugs are in contact with swine food sources needs further investigation (Pereira et al. 2010).

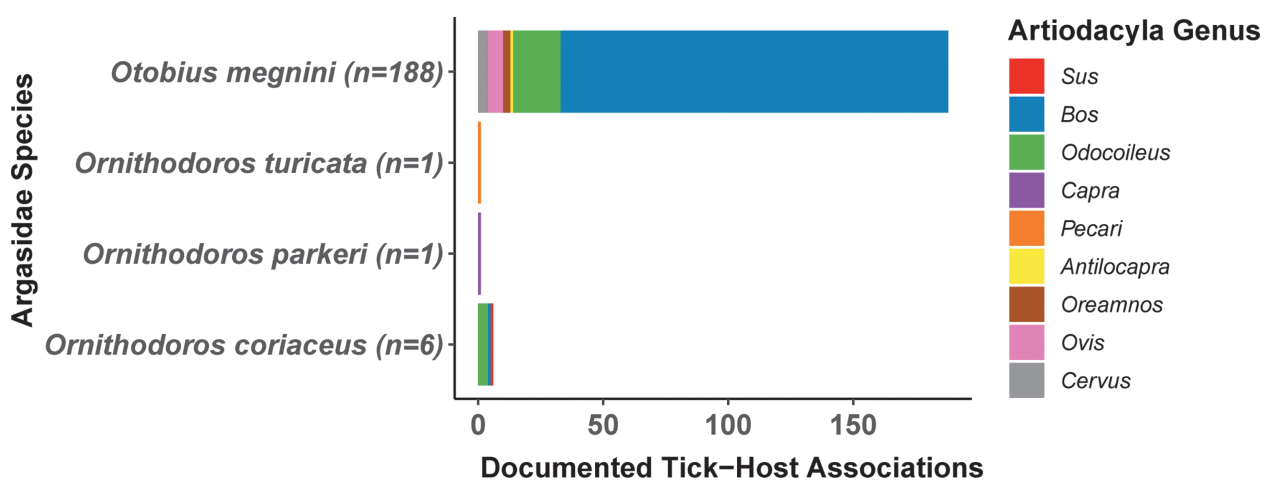

FIG. 2. The association of Argasid ticks in the United States with vertebrate hosts in the Artiodactyla order by taxonomic genera. Soft tick (Argasidae) collection records across the United States from 1891 to 2004 were compiled from the U.S. National Tick Collection. Data represent the number of unique collections of each soft tick species that were associated with hosts from different Artiodactyla genera (Antilocapra, Bos, Capra, Cervus, Odocoileus, Oreamnos, Ovis, Pecari, Sus). 


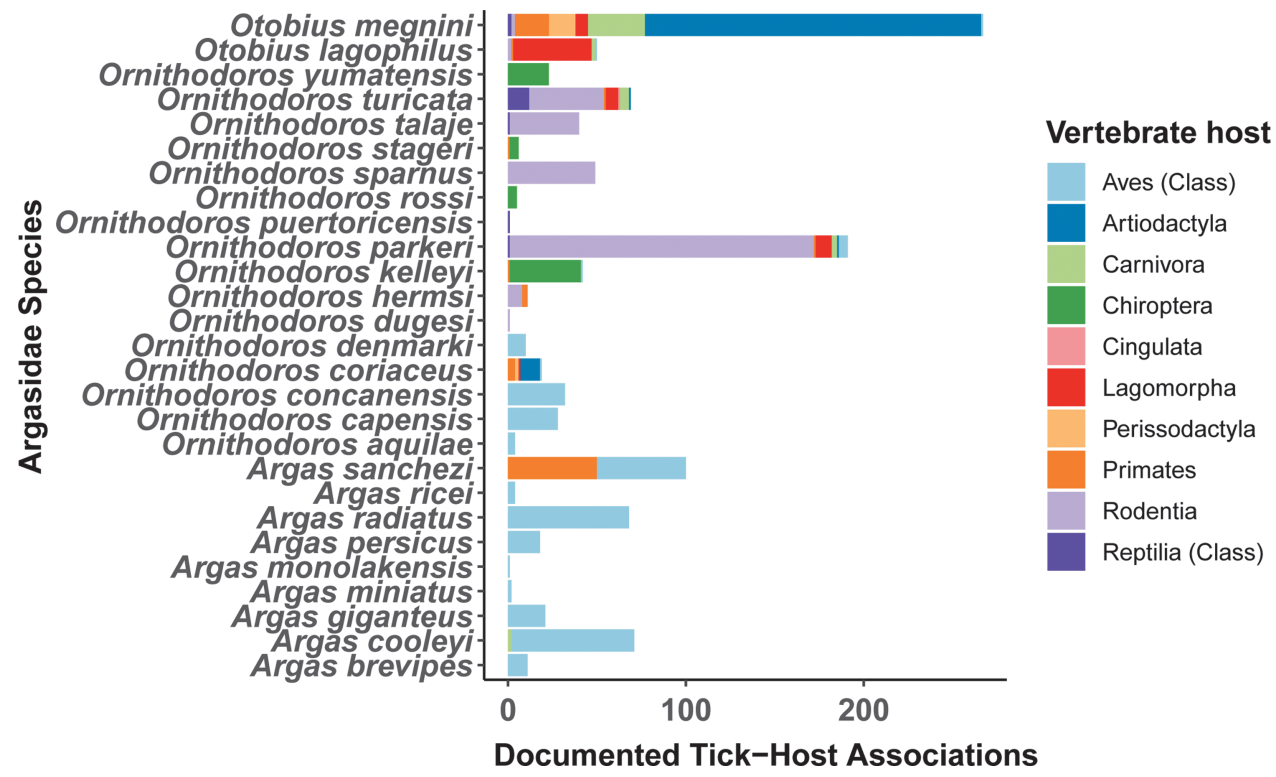

FIG. 3. The association of Argasid ticks with vertebrate host orders in the United States. Soft tick (Argasidae) collection records across the United States from 1891 to 2004 were compiled from the U.S. National Tick Collection. Data represent the number of unique collections of each soft tick species that were associated with hosts from different mammalian orders (Rodentia, Cingulata, Carnivora, Chiroptera, Lagomorpha, Artiodactyla, Perissodactyla, and Primates). Vertebrate host taxa from the taxonomic groups of Aves and Reptilia are only documented at the resolution of Class.
Overall, the capacity for arthropods to transmit the virus as biological vectors during a subsequent feeding event appears to be restricted to soft tick vectors in the genus Ornithodoros. In particular, only three species in the U.S. have demonstrated an ability to transmit ASFV: O. coriaceus, O. puertoricensis, and O. turicata (Table 1). However, transmission data for $O$. coriaceus and $O$. turicata were limited and experimental replicates should be completed to better understand transmission efficiency (Hess et al. 1987).

Further studies should focus on the unique aspects of soft tick biology and how this may influence ASFV transmission ecology. Soft ticks are known to survive for multiple decades as adults (Endris and Hess 1992), a unique trait that may afford ASFV a mechanism to persist in nature should vectors remain infectious. Furthermore, many soft tick vectors in the Ornithodoros genus feed multiple times through development, sometimes feeding as much as once every 3 days on multiple hosts, including other engorged ticks (Butler and Gibbs 1984). Experimental observations also demonstrate ASFV can persist for up to 588 days in some vector species, can be sexually transmitted between vectors, and vertically transmitted from adults to offspring (Endris and Hess 1992).

These biological characteristics highlight an incredible capacity for soft tick vectors to function as a viral reservoir and stress a need to understand soft tick biology in the U.S. to proactively identify and prevent mechanisms of ASFV establishment. Even though ASFV may be maintained through transovarial, sexual, and transtadial transmission, studies demonstrate infected $O$. erraticus and $O$. moubata colonies have cleared infections when reared on noninfectious blood suggesting there are limits to the long-term capacity of $\mathrm{Or}$ nithodoros ticks to function as viral reservoirs (Hess et al. 1989). Overall, the vector competence of most soft tick species in the U.S. remains unknown (Table 2) and additional vector competence studies are warranted. Future transmission experiments should focus on soft tick vectors that associate with competent hosts.

Numerous studies demonstrated that animals from the family Suidae are competent hosts, and there is a lack of experimental or epidemiological evidence to implicate any other mammalian taxa as amplification hosts. The experimental infection studies we reviewed show Suids are extremely susceptible to multiple strains of ASFV and can contract the virus through various modes of transmission, including direct tissue contact, fomites, aerosols, and vectors. Infection dynamics demonstrate that S. scrofa (domestic and feral pigs) produce relatively high viremic titers compared with sylvatic hosts found in endemic regions (bushpigs and warthogs) (Fig. 1). High viremia may contribute to pig-to-pig transmission in domestic swine operations; however, high contact rates in rearing operations likely play an important role (Wilkinson et al. 1977, Guinat et al. 2014).

Furthermore, it appears that Suids surviving clinical disease can support persistent viremic infections, in some cases for up to 70 days (Anderson et al. 1998, Ferreira et al. 2012). Assuming ASFV transmission is dependent on viremia levels, waning virus titers suggest that hosts become less infectious over time and, barring mechanisms of viral recrudescence or vertical transmission, persistently infected hosts may reach a point where they are no longer infectious. However, because most studies are terminated due to ethical or economic constraints, understanding the long-term dynamics of ASFV viremia continues to be a challenge.

A scarcity of biological and experimental data for most soft tick species in the U.S. makes it difficult to assess which vectors pose a risk for contributing to ASFV transmission. Knowledge of soft tick-host utilization and relative abundance can help identify which soft tick species are good candidates for vector competence studies. Contact rates are important in determining the potential contribution of organisms to pathogen transmission. Rates of contact between vertebrates and vectors can be difficult to assess in a natural setting, especially for Ornithodoros vectors that feed in a matter of minutes (Butler and Gibbs 1984). Molecular tools used to identify residual traces of host DNA in abdomens of vectors can be of use, but life history traits and trapping strategies have limited advancements in this area of soft tick ecology. 


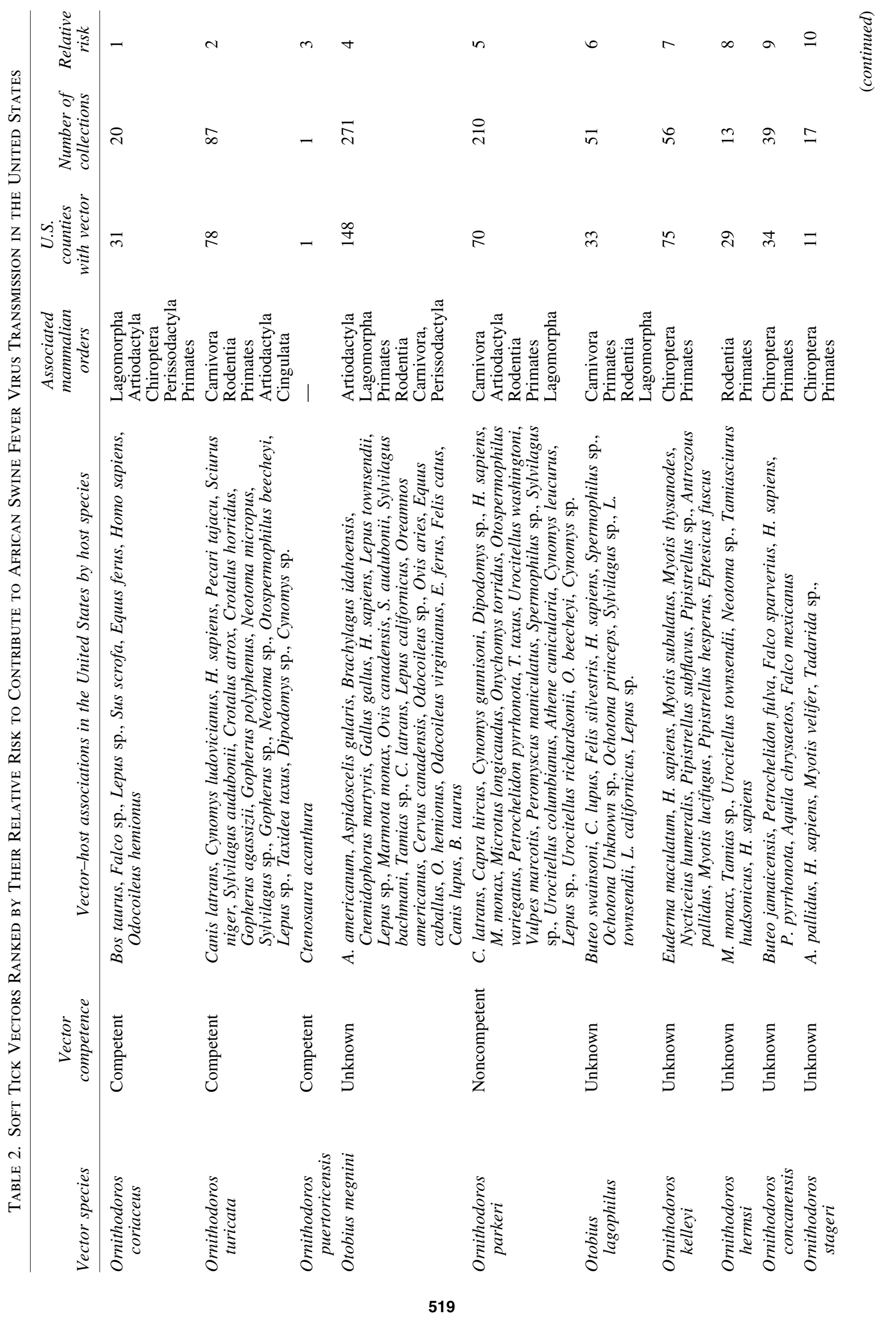




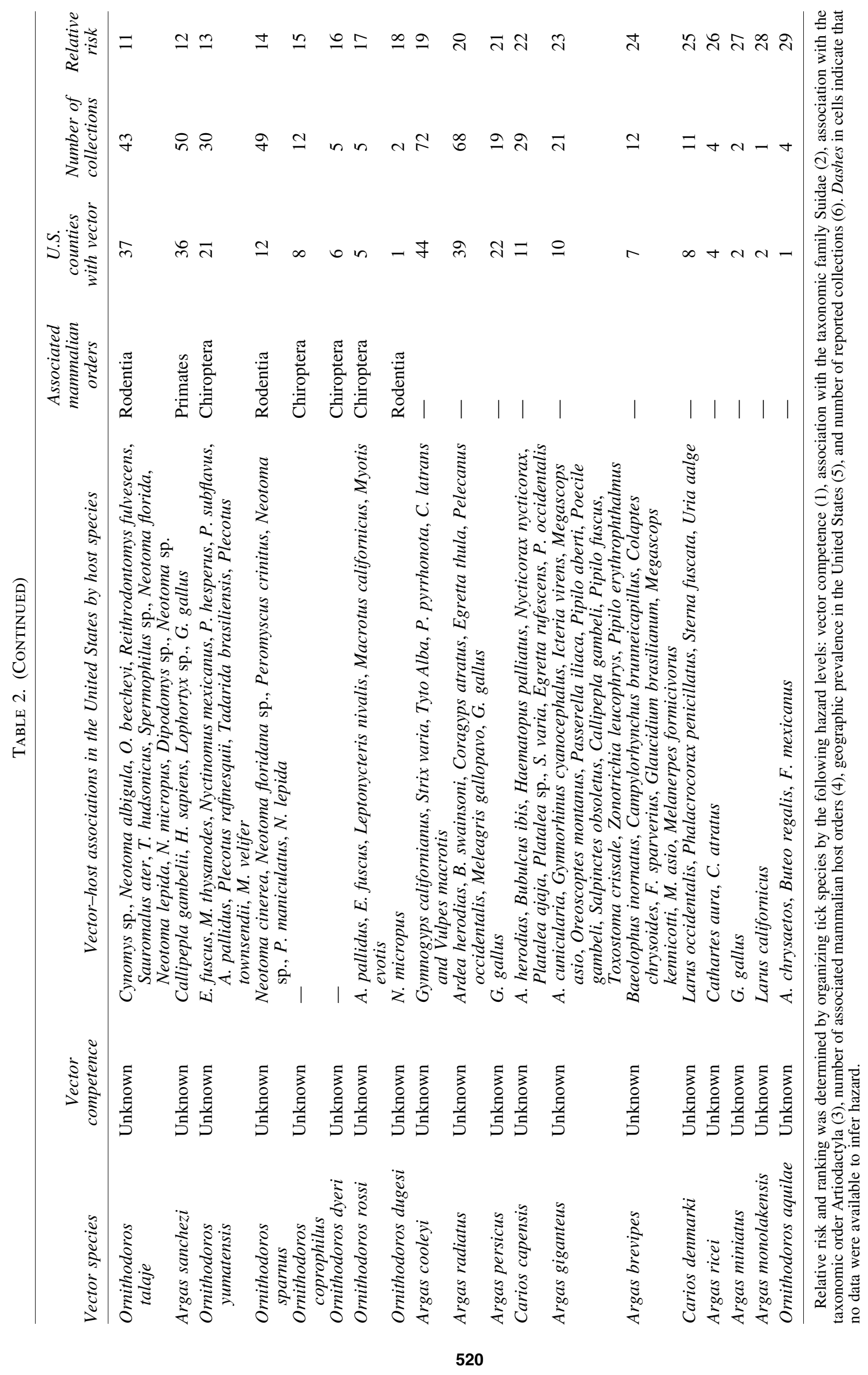


To overcome the fact that host association data remain underdeveloped for the majority of soft tick species in the U.S., we used metadata from the US National Tick Collection, which documents vertebrate hosts present in the area of collection to estimate tick-host contact. In this dataset, only a single soft tick species-O. coriaceus-was documented to be associated with swine in the U.S. Some tick vectors were associated with the sister taxa from the Artiodactyla order (Figs. 2, 3, and Table 2), including O. megnini with 190 records of being associated with Artiodactyla, suggesting this vector may persist in high abundance in domestic or peridomestic settings where livestock are held. In this context, high abundance may lead to incidental parasitism of domestic or peridomestic swine, although such parasitism has not been documented.

Overall, the lack of evidence demonstrating interactions between soft ticks and swine emphasizes a need to better understand which soft tick species interact with swine in the U.S. Prior studies demonstrate feral swine across Texas are infested with a variety of hard ticks, including with A. americanum, A. mixtum, A. maculatum, Dermacentor albipictus, Dermacentor halli, Dermacentor variablis, and Ixodes scapularis (Coombs and Springer 1974, Sanders et al. 2013). Although these efforts were quite extensive, infestations by Argasid ticks were not documented, including $O$. megnini despite the concentrated inspection of the ears where these parasites are known to commonly attach. Until intensive fieldwork across the U.S. updates our understanding of soft tick-swine interactions, we can utilize results generated by this synthesis to estimate the likelihood of tickswine interactions.

Besides $O$. coriaceus, which has been documented to associate with swine, results from this analysis suggest $O$. megnini, $O$. parkeri, and $O$. turicata are the most likely soft tick species to associate with the family Suidae because of their association with the order Artiodactyla (Figs. 2 and $3)$. Soft ticks that associate with mammals (O. lagophilus, O. kelleyi, O. hermsi, O. concanensi, O. stageri, O. talaje, and A. sanchezi, O. yumatensis, O. sparnus, A. coprophilus, $O$. dyeri, O. rossi, A. cooleyi, and $O$. dugesi) are also potential candidates to utilize swine as hosts (Table 2).

\section{Conclusion}

Our analysis highlights several gaps in knowledge for which additional research could enhance predictions of important hosts and vectors for ASFV and better inform proactive prevention and management strategies.

Overall, PCR is a useful diagnostic tool, however, molecular PCR-based methods for assessing virulence in experimental infections may result in overestimates of vector or host competence considering ASFV is a DNA virus capable of persisting as noninfectious nucleic acid (Basto et al. 2006b, Guinat et al. 2014). When possible, viral titrations should be performed in swine macrophage media and quantified by assessing 50\% endpoints through hemadsorption to assess competence (Reed and Muench 1938, Plowright et al. 1968). Additionally, future vector transmission studies should make an effort to evaluate vector survivorship, vertical transmission, viral persistence, and alternative routes of transmission (e.g., oral transmission) for soft tick species found in North America.
Similarly, host competence studies should try to evaluate dose-dependent transmission thresholds for vector transmission and horizontal transmission. Transmission thresholds and various routes of transmission appear to play an important role in ASFV ecology and epidemiology demonstrating the ability for ASFV to persist in a variety of ecological contexts (such as the absence of tick vectors) (Guinat et al. 2016a, 2016b, Pietschmann et al. 2016). However, knowledge on the impacts of mechanisms such as viral recrudescence or vertical transmission on viral maintenance remains underdeveloped (Anderson et al. 1998, Guinat et al. 2016a, Post et al. 2017).

Furthermore, even though evidence suggests hard ticks are not biological vectors of the virus (Ferreira et al. 2014), as a precautionary study, it may be necessary to test the vector competence of hard ticks associated with swine in the U.S. such as A. maculatum, A. mixtum, Dermacentor variabilis, or Amblyomma tenellum (Cohen et al. 2010, Corn et al. 2016). Finally, intensive field work across the U.S., where swine hosts persist, is necessary to incriminate species of soft ticks interacting with competent hosts in the U.S. and identify hot spots for potential ASFV establishment; this objective would be facilitated by enhanced bloodmeal analysis techniques to identify the vertebrate hosts upon which soft tick vectors have fed days, weeks, or years earlier.

No treatment or vaccine exists to prevent or combat ASFV infection, and subsequently, disease management is focused on intensive surveillance, restricting contact between wild and domestic swine, vector control, and policies that combat risky swill practices (Bellini et al. 2016, Halasa et al. 2016). In countries free of ASFV, such as the U.S., the importation of live pigs and pork products from infected areas is banned. However, if introduced to the U.S., the success of eradication relies on early detection, mass killing of infected animals, vector control, and proper disinfection. In this study, we demonstrate that $O$. coriaceus, $O$. turicata, and $O$. puertoricensis are high-risk vectors capable of contributing to ASFV transmission should the virus be introduced in the U.S. Accordingly, control and surveillance strategies in the U.S. can be tailored to the biology of these ticks.

\section{Acknowledgments}

The authors thank Dr. Lorenza Beati and Colleen Evans at the U.S. National Tick Collection for providing soft tick records. They thank Cierra Briggs and Abraham Munnerlyn for assistance in data entry. Financial support was provided by the Department of Homeland Security (DHS) Science and Technology Directorate (S\&T) under agreement \#HSHQDC17-C-B0003.

\section{Author Disclosure Statement}

No competing financial interests exist.

\section{References}

Anderson E, Hutchings G, Mukarati N, Wilkinson P. African swine fever virus infection of the bushpig (Potamochoerus porcus) and its significance in the epidemiology of the disease. Vet microbiol 1998; 62:1-15.

Argilaguet JM, Perez-Martin E, Nofrarias M, Gallardo C, et al. DNA vaccination partially protects against African swine 
fever virus lethal challenge in the absence of antibodies. PLoS One 2012; 7:e40942.

Arias M, Sánchez-Vizcaíno JM. Chapter 4.1: African swine fever. In: Trends in emerging viral infections of swine. Iowa State Press, Ames, Iowa, 2002.

Basto AP, Nix RJ, Boinas F, Mencles S, et al. Kinetics of African swine fever virus infection in Ornithodoros erraticus ticks. J Gen Virol 2006a; 87:1863-1871.

Basto AP, Portugal RS, Nix RJ, Cartaxeiro C, et al. Development of a nested PCR and its internal control for the detection of African swine fever virus (ASFV) in Ornithodoros erraticus. Arch Virol 2006b; 151:819-826.

Bellini S, Rutili D, Guberti V. Preventive measures aimed at minimizing the risk of African swine fever virus spread in pig farming systems. Acta Vet Scand 2016; 58:82.

Boinas FS, Wilson AJ, Hutchings GH, Martins C, et al. The persistence of African swine fever virus in field-infected Ornithodoros erraticus during the ASF endemic period in Portugal. PLoS One 2011; 6:e20383.

Brown VR, Bevins SN. A review of African swine fever and the potential for introduction into the United States and the possibility of subsequent establishment in feral swine and native ticks. Front Vet Sci 2018; 5:11.

Butler J, Gibbs E. Distribution of potential soft tick vectors of African swine fever in the Caribbean region (Acari: Argasidae). Prev Vet Med 1984; 2:63-70.

Carlson J, O’Donnell V, Alfano M, Velazquez Salinas L, et al. Association of the host immune response with protection using a live attenuated African swine fever virus model. Viruses 2016; 8:291-306.

Cohen S, Freye J, Dunlap B, Dunn J, et al. Host associations of Dermacentor, Amblyomma, and Ixodes (Acari: Ixodidae) ticks in Tennessee. J Med Entomol 2010; 47:415-420.

Coombs DW, Springer MD. Parasites of feral pig X European wild boar hybrids in southern Texas. J Wildl Dis 1974; 10: 436-441.

Corn JL, Duhaime RA, Alfred JT, Mertins JW, et al. Survey for ticks on feral swine within a cattle fever tick-infested landscape in Texas, USA. Syst Appl Acarol 2016; 21:1564-1570.

Costard S, Jones BA, Martinez-Lopez B, Mur L, et al. Introduction of African swine fever into the European union through illegal importation of pork and pork products. PLoS One 2013; 8:e61104.

Costard S, Wieland B, De Glanville W, Jori F, et al. African swine fever: How can global spread be prevented? Philos Trans R Soc Lond B Biol Sci 2009; 364:2683-2696.

Davies K, Goatley LC, Guinat C, Netherton CL, et al. Survival of African swine fever virus in excretions from pigs experimentally infected with the Georgia 2007/1 isolate. Transbound Emerg Dis 2017; 64:425-431.

de Carvalho Ferreira H, Weesendorp E, Quak S, Stegeman J, et al. Quantification of airborne African swine fever virus after experimental infection. Vet microbiol 2013; 165:243251.

Endris RG, Haslett TM, Hess WR. Experimental transmission of African swine fever virus by the tick Ornithodoros (Alectorobius) puertoricensis (Acari, Argasidae). J Med Entomol 1991; 28:854-858.

Endris RG, Hess WR. Experimental transmission of African swine fever virus by the soft tick Ornithodoros (Pavlovskyella) marocanus (Acari, Ixodoidea, Argasidae). J Med Entomol 1992; 29:652-656.

Endris RG, Hess WR, Caiado JM. African swine fever virusinfection in the Iberian soft tick, Ornithodoros (Pavlovs- kyella) marocanus (Acari, Argasidae). J Med Entomol 1992; 29:874-878.

Ewald PW. Transmission modes and evolution of the parasitism-mutualism continuum. Ann N Y Acad Sci 1987; 503:295-306.

Fasina FO, Lazarus DD, Spencer BT, Makinde AA, et al. Cost implications of African swine fever in smallholder farrow-tofinish units: Economic benefits of disease prevention through biosecurity. Transbound Emerg Dis 2012; 59:244-255.

Fernandez A, Williams D. Eradication of African swine fever in Cuba. In: Animal Health in the Americas, 1979. Proceedings of the XII Inter-American Meeting on foot-and-mouth disease and zoonoses control, Curacao, 17-20 April 1979. Pan American Health Organization, Washington D.C. 1980:60-65.

Ferreira H, Weesendorp E, Elbers ARW, Bouma A, et al. African swine fever virus excretion patterns in persistently infected animals: A quantitative approach. Vet Microbiol 2012; 160:327-340.

Ferreira HCD, Backer JA, Weesendorp E, Klinkenberg D, et al. Transmission rate of African swine fever virus under experimental conditions. Vet Microbiol 2013; 165:296-304.

Ferreira HCD, Zuquetec ST, Wijnveld M, Weesendorp E, et al. No evidence of African swine fever virus replication in hard ticks. Ticks Tick Borne Dis 2014; 5:582-589.

Galindo-Cardiel I, Ballester M, Solanes D, Nofrarias M, et al. Standardization of pathological investigations in the framework of experimental ASFV infections. Virus Res 2013; 173: 180-190.

Gallardo C, Soler A, Nieto R, Cano C, et al. Experimental infection of domestic pigs with African swine fever virus Lithuania 2014 Genotype II Field Isolate. Transbound Emerg Dis 2017; 64:300-304.

GBIF.org. GBIF Occurrence Download. Available at https://doi .org/10.15468/dl.1pdxff 2018

Genovesi E, Knudsen R, Whyard T, Mebus C. Moderately virulent African swine fever virus infection: Blood cell changes and infective virus distribution among blood components. Am J Trop Med 1988; 49:338-344.

Gervasi SS, Civitello DJ, Kilvitis HJ, Martin LB. The context of host competence: A role for plasticity in host-parasite dynamics. Trends Parasitol 2015; 31:419-425.

Gogin A, Gerasimov V, Malogolovkin A, Kolbasov D. African swine fever in the North Caucasus region and the Russian Federation in years 2007-2012. Virus Res 2013; 173:198-203.

Golnar AJ, Turell MJ, LaBeaud AD, Kading RC, et al. Predicting the mosquito species and vertebrate species involved in the theoretical transmission of Rift Valley fever virus in the United States. PLoS Negl Trop Dis 2014; 8:e3163.

Groocock CM, Hess WR, Gladney WJ. Experimental transmission of African swine fever virus by Ornithodoros coriaceus, an Argasid tick indigenous to the United States. Am J Vet Res 1980; 41:591-594.

Guglielmone AA, Robbins RG, Apanaskevich DA, Petney TN, et al. Argasidae, Ixodidae and Nuttalliellidae (Acari: Ixodida) of the world: A list of valid species names. Zootaxa 2010; 2528:1-28.

Guinat C, Gogin A, Blome S, Keil G, et al. Transmission routes of African swine fever virus to domestic pigs: Current knowledge and future research directions. Vet Rec 2016a; 178:262-267.

Guinat C, Gubbins S, Vergne T, Gonzales JL, et al. Experimental pig-to-pig transmission dynamics for African swine fever virus, Georgia 2007/1 strain. Epidemiol Infect 2016b; 144:3564-3566. 
Guinat C, Reis AL, Netherton CL, Goatley L, et al. Dynamics of African swine fever virus shedding and excretion in domestic pigs infected by intramuscular inoculation and contact transmission. Vet Res 2014; 45:93.

Halasa T, Botner A, Mortensen S, Christensen H, et al. Control of African swine fever epidemics in industrialized swine populations. Vet Microbiol 2016; 197:142-150.

Hamer GL, Kitron UD, Goldberg TL, Brawn JD, et al. Host selection by Culex pipiens mosquitoes and West Nile virus amplification. Am J Trop Med 2009; 80:268-278.

Hess W, Endris R, Haslett T, Monahan M, et al. Potential arthropod vectors of African swine fever virus in North America and the Caribbean basin. Vet Parasitol 1987; 26: 145-155.

Hess WR, Endris RG, Lousa A, Caiado JM. Clearance of African swine fever virus from infected tick (Acari) colonies. J Med Entomol 1989; 26:314-317.

Johnston SL, Wellens K, Siegel C. Comparison of hemagglutination and hemadsorption tests for influenza detection. Diagn Microbiol Infect Dis 1992; 15:363-365.

Jori F, Bastos AD. Role of wild suids in the epidemiology of African swine fever. Ecohealth 2009; 6:296-310.

Karalyan Z, Zakaryan H, Sargsyan K, Voskanyan H, et al. Interferon status and white blood cells during infection with African swine fever virus in vivo. Vet Immunol Immunopathol 2012; 145:551-555.

Kilpatrick AM, Daszak P, Jones MJ, Marra PP, et al. Host heterogeneity dominates West Nile virus transmission. Philos Trans R Soc Lond B Biol Sci 2006; 273:2327-2333.

Kleiboeker SB, Burrage TG, Scoles GA, Fish D, et al. African swine fever virus infection in the argasid host, Ornithodoros porcinus porcinus. J Virol 1998; 72:1711-1724.

Knudsen RC, Genovesi EV. In vivo and in vitro effects of moderately virulent African swine fever virus on mitogenesis of pig lymphocytes. Vet Immunol Immunopathol 1987; 15 : 323-336.

Komar N, Langevin S, Hinten S, Nemeth N, et al. Experimental infection of North American birds with the New York 1999 strain of West Nile virus. Emerg Infect Dis 2003; 9:311.

McVicar JW. Quantitative aspects of the transmission of African swine fever. Am J Vet Res 1984; 45:1535-1541.

Mellor PS, Kitching RP, Wilkinson PJ. Mechanical transmission of Capripox virus and African Swine fever virus by Stomoxys calcitrans. Res Vet Sci 1987; 43:109-112.

Mellor PS, Wilkinson PJ. Experimental transmission of African swine fever virus by Ornithodoros savignyi (Audouin). Res Vet Sci 1985; 39:353-356.

Montgomery RE. On a form of swine fever occurring in British East Africa (Kenya Colony). J Comp Pathol Ther 1921; 34: 159-191.

Moura J, McManus C, Bernal F, De Melo C. An analysis of the 1978 African swine fever outbreak in Brazil and its eradication. Rev Sci Tech 2010; 29:549.

Mur L, Igolkin A, Varentsova A, Pershin A, et al. Detection of African swine fever antibodies in experimental and field samples from the Russian Federation: Implications for control. Transbound Emerg Dis 2016; 63:E436-E440.

Nieto-Pelegrin E, Rivera-Arroyo B, Sanchez-Vizcaino JM. First detection of antibodies against African swine fever virus in faeces samples. Transbound Emerg Dis 2015; 62:594-602.

O'Donnell V, Holinka LG, Gladue DP, Sanford B, et al. African swine rever virus Georgia isolate harboring deletions of MGF360 and MGF505 genes is attenuated in swine and confers protection against challenge with virulent parental virus. J Virol 2015a; 89:6048-6056.

O’Donnell V, Holinka LG, Krug PW, Gladue DP, et al. African swine fever virus Georgia 2007 with a deletion of virulenceassociated gene 9GL (B119L), when administered at low doses, leads to virus attenuation in swine and induces an effective protection against homologous challenge. J Virol 2015b; 89:8556-8566.

O'Donnell V, Tiolinka LG, Sanford B, Krug PW, et al. African swine fever virus Georgia isolate harboring deletions of 9GL and MGF360/505 genes is highly attenuated in swine but does not confer protection against parental virus challenge. Virus Res 2016; 221:8-14.

Pereira KS, Schmidt FL, Barbosa RL, Guaraldo AM, et al. Transmission of Chagas disease (American trypanosomiasis) by food. Adv Food Nutr Res 2010; 59:63-85.

Pietschmann J, Guinat C, Beer M, Pronin V, et al. Course and transmission characteristics of oral low-dose infection of domestic pigs and European wild boar with a Caucasian African swine fever virus isolate. Arch Virol 2015; 160: 1657-1667.

Pietschmann J, Mur L, Blome S, Beer M, et al. African swine fever virus transmission cycles in Central Europe: Evaluation of wild boar-soft tick contacts through detection of antibodies against Ornithodoros erraticus saliva antigen. BMC Vet Res 2016; $12: 1$.

Plowright W, Parker J, Staple RF. Growth of a virulent strain of African swine fever virus in domestic pigs. J Hyg 1968; 66: 117-134.

Plowright W, Perry C, Greig A. Sexual transmission of African swine fever virus in the tick, Ornithodoros moubata porcinus, Walton. Res Vet Sci 1974; 17:106.

Plowright W, Perry CT, Peirce MA, Parker J. Experimental infection of Argasid tick, Ornithodoros moubata porcinus, with African swine fever virus. Arch Gesamte Virusforsch 1970; 31:33-50.

Popescu L, Gaudreault NN, Whitworth KM, Murgia MV, et al. Genetically edited pigs lacking CD163 show no resistance following infection with the African swine fever virus isolate, Georgia 2007/1. Virology 2017; 501:102-106.

Post J, Weesendorp E, Montoya M, Loeffen WL. Influence of Age and Dose of African swine fever virus infections on clinical outcome and blood parameters in pigs. Viral Immunol 2017; 30:58-69.

ProMED-mail. African swine fever-Asia (15): China (Liaoning) Domestic Swine, Follow-up, OIE. ProMED-Mail 2018; 20181006.6074582. Accessed October 15, 2018.

Ramiroibanez F, Escribano JM, Alonso C. Application of a monoclonal-antibody recognizing protein P-30 to detect African swine fever virus-infected cells in peripheral blood. J Virol Methods 1995; 55:339-345.

Reed LJ, Muench H. A simple method of estimating fifty per cent endpoints. Am J Epidemiol 1938; 27:493-497.

Reis AL, Abrams CC, Goatley LC, Netherton C, et al. Deletion of African swine fever virus interferon inhibitors from the genome of a virulent isolate reduces virulence in domestic pigs and induces a protective response. Vaccine 2016; 34:4698-4705.

Rennie L, Wilkinson PJ, Mellor PS. Effects of infection of the tick Ornithodoros moubata with African swine fever virus. Med Vet Entomol 2000; 14:355-360.

Ribeiro R, Otte J, Madeira S, Hutchings GH, et al. Experimental infection of Ornithodoros erraticus sensu stricto with two portuguese African swine fever virus strains. Study of factors 
involved in the dynamics of infection in ticks. PLoS One 2015; 10:e0137718.

Roelandt S, Van der Stede Y, D'Hondt B, Koenen F. The Assessment of African swine fever virus risk to Belgium early 2014, using the quick and semiquantitative pandora screening protocol. Transbound Emerg Dis 2017; 64:237249.

Roellig DM, Ellis AE, Yabsley MJ. Oral transmission of Trypanosoma cruzi with opposing evidence for the theory of carnivory. J Parasitol 2009; 95:360-364.

Sanchez-Cordón PJ, Montoya M, Reis A, et al. African swine fever: A re-emerging viral disease threatening the global pig industry. Veterinary J 2018; 233:41-48.

Sanders DM, Schuster AL, McCardle PW, Strey OF, et al. Ixodid ticks associated with feral swine in Texas. J Vector Ecol 2013; 38:361-373.

Sanford B, Holinka LG, O'Donnell V, Krug PW, et al. Deletion of the thymidine kinase gene induces complete attenuation of the Georgia isolate of African swine fever virus. Virus Res 2016; 213:165-171.

Thomson GR, Gainaru MD, Van Dellen AF. Experimental infection of warthos (Phacochoerus aethiopicus) with African swine fever virus. Onderstepoort J Vet Res 1980; 47: 19-22.

United States Department of Agriculture. GAIN Report: Russia 2017 Livestock and Products Annual RS1757. Global Agricultural Information Network 2017.

Villeda CJ, Williams SM, Wilkinson PJ, Vinuela E. Hemostatic abnormalities in African swine fever-A comparison of 2 virus strains of different virulence (Dominican Republic 78 and Malta 78). Arch Virol 1993; 130:71-83.
Wilkinson P, Donaldson A. Transmission studies with African swine fever virus: The early distribution of virus in pigs infected by airborne virus. J Comp Path 1977; 87:497-501.

Wilkinson P, Wardley R, Williams S. African swine fever virus (Malta/78) in pigs. J Comp Path 1981; 91:277-284.

Wilkinson PJ, Donaldson AI, Greig A, Bruce W. Transmission studies with African swine fever virus-infections of pigs by airborne virus. J Comp Path 1977; 87:487-495.

Wooldridge M, Hartnett E, Cox A, Seaman M. Quantitative risk assessment case study: Smuggled meats as disease vectors. Rev Sci Tech 2006; 25:105-117.

Address correspondence to: Gabriel L. Hamer

Texas A\&M AgriLife Research

Department of Entomology Texas A\&M University

Heep Center Room 515

2475 TAMU

College Station, TX 77843

E-mail: ghamer@tamu.edu

Andrew Golnar

Texas A\&M AgriLife Research

Department of Entomology

Texas A\&M University

Heep Center Room 515

2475 TAMU

College Station, TX 77843

E-mail: agolnar@tamu.edu 